\title{
Determinants of polyunsaturated fatty acid concentrations in erythrocytes of pregnant Japanese women from a birth cohort study: study protocol and baseline findings of an adjunct study of the Japan environment \& Children's study
}

Shoji Saito ${ }^{1,2^{*}}$ (D) Terue Kawabata ${ }^{3}$, Nozomi Tatsuta ${ }^{4}$, Fumiko Kimura ${ }^{5,6}$, Teruo Miyazawa ${ }^{6,7}$, Satoshi Mizuno ${ }^{1}$, Hidekazu Nishigori ${ }^{1}$, Takahiro Arima ${ }^{8}$, Yasuo Kagawa ${ }^{4}$, Kouichi Yoshimasu $^{9}$, Kanami Tsuno ${ }^{9}$, Yuki Ito ${ }^{10}$, Michihiro Kamijima ${ }^{10}$, Kunihiko Nakai ${ }^{4}$, Nobuo Yaegashi ${ }^{1}$ and Miyagi Study Group of Japan Environment \& Children's Study

\begin{abstract}
Background: N-3 polyunsaturated fatty acids ( $n-3$ PUFA) may have multiple beneficial effects on the outcome of pregnancy, maternal health and child development. The present study introduced the protocol of a birth cohort study to examine the beneficial effects of n-3 PUFA status in pregnant Japanese women as an adjunct study of the Japan Environment and Children's Study (JECS).

Methods: The JECS participants in the coastal areas of Miyagi Prefecture were further invited to participate in this adjunct study, and 1,878 pregnant women were enrolled prior to delivery. Their n-3 PUFA status was evaluated with fatty acid profiles in erythrocytes of maternal blood collected from 1,623 mothers at 24-30 weeks of gestation and cord blood from 1,505 deliveries.

Results: The baseline results, including comprehensive data on the fatty acid status and determinants affecting the PUFA status, were analyzed. In stepwise multivariate analyses, the cord blood docosahexaenoic acid (DHA) level was found to be significantly influenced by the DHA level in maternal blood, the child's sex, and the gestational period. The maternal DHA level was influenced by fish intake, maternal age, and the prepregnancy body mass index. While cord blood eicosapentaenoic acid (EPA) was influenced by maternal EPA, fish intake, and season at birth, additional factors such as maternal education, household income, and smoking habits affected the maternal EPA content.
\end{abstract}

Conclusion: Further studies are warranted to clarify the nutritional impacts of n-3 PUFA in pregnant Japanese women of the cohort study.

Keywords: Birth cohort study, Docosahexaenoic acid, Eicosapentaenoic acid, Japan environment and children's study, N-3 polyunsaturated fatty acids

\footnotetext{
* Correspondence: shojisaiko5324@yahoo.co.jp

'Department of Gynecology and Obstetrics, Tohoku University Graduate

School of Medicine, Sendai, Japan

${ }^{2}$ Department of Obstetrics and Gynecology, Yamagata Prefectural Central

Hospital, Aoyagi 1800, Yamagata 990-2292, Japan

Full list of author information is available at the end of the article
} 


\section{Background}

N-3 polyunsaturated fatty acids (n-3 PUFA) are of critical importance during pregnancy and early life. Several observational studies and randomized trials showed the beneficial effects of n-3 PUFA during pregnancy and/or lactation for normal fetal and postnatal child growth and development such as cognitive performance, attention, information processing, and visual acuity [1-6]. Furthermore, PUFA during early life may modify fetal programming that influences the susceptibility and individual risk for developing metabolic diseases throughout life [7]. Supplementation of n-3 PUFA during pregnancy reduces the incidence of early preterm birth [6]. The maternal n-3 PUFA status has also been shown to be associated with maternal outcomes such as psychological distress [8] and postpartum depression [9]. Maternal obesity and excessive weight gain during pregnancy are reported to be associated with an adverse fatty acid profile such as higher concentrations of saturated fatty acids, monounsaturated fatty acids, and n-6 PUFA in plasma of pregnant women [10]. Although the causal relation with fatty acid intake remains undetermined, these findings suggest the importance of the quality of the fatty acid profile in pregnant women.

Since fish and seafood are rich in n-3 PUFA, fish consumption is the primary route for the intake. Therefore, the n-3 PUFA level in the blood of fish-eating populations is usually high. However, most of the above studies indicating beneficial effects have been conducted in the USA or other Western countries where most women have relatively low intakes of n-3 PUFA [11]. In this context, it should be examined whether n-3 PUFA is still beneficial in a population that consumes a large amount of fish and seafood.

Furthermore, fish are also contaminated with several toxic chemicals such as methylmercury and persistent organic pollutants (POPs) through bioaccumulation in the aquatic food chain. Prenatal exposure to these chemicals induces several adverse effects, including fetal growth impairment $[12,13]$ and delayed neurobehavioral development $[14,15]$. These findings suggest the necessity of mutual adjustment of both factors, and the balance of the beneficial and adverse effects of fish consumption should be considered to explore the nutritional benefits of $n-3$ PUFA in pregnancy for fish-eating populations, including Japanese women.

The PUFA status of the fetus and infant depends primarily on the maternal PUFA status and has to be ensured by placental [4] or breast milk transfer [16]. The maternal PUFA status is primarily dependent on maternal fish consumption, and several other factors have been shown to be related to the fatty acid status in pregnant women. These include the birth order [17], prepregnancy body mass index (BMI) [10], period of gestation [18], maternal gestational weight gain [10], smoking habit [11, 18], and
PUFA supplementation [18]. In healthy adults, additional factors, including age [19-21], BMI [19], educational level [22, 23], income [22], race [20], alcohol use [20], and hormonal status [24] were also shown to affect the PUFA profile, and physical activity has been identified to be inversely associated in Japanese [21]. While eicosapentaenoic acid (EPA, 20:5n-3), docosahexaenic acid (DHA, 22:6n-3) and arachidonic acid (ARA, 20:4n-6) can be formed from the precursors $\alpha$-linolenic acid (ALA, 18:3n-3) and linoleic acid (LA, 18:2n-6), respectively [25], several single nucleotide polymorphisms (SNPs) in the fatty acid desaturase gene cluster affect the PUFA profile in Japanese women [26]. A better knowledge of these factors could help to improve the PUFA status in pregnant women.

To clarify the beneficial effects of PUFA on maternal outcomes and child development in pregnant Japanese women, we designed a birth cohort study as an adjunct study of The Japan Environment \& Children's Study (JECS). JECS is an ongoing national large prospective cohort study to evaluate the impacts of various environmental factors on child health and development. As the adjunct study, we added the determination of PUFA levels in maternal and cord blood erythrocytes and maternal breast milk to evaluate the nutritional advantages of maternal PUFA intake. In this interim report, we present the study protocol, participants' characteristics, the baseline data and the determinants of PUFA status in maternal and cord blood erythrocytes.

\section{Methods}

\section{Study population}

JECS, funded by the Ministry of the Environment (MOE), is a large, nationwide longitudinal birth cohort study with follow-up programs to examine health from the early stages of pregnancy until the participating child reaches 13 years of age. Details of the JECS recruitment and sampling strategy as well as data collection procedures have been previously described [27]. Of the 15 regional centers of JECS, the Miyagi Regional Center covers 14 local government areas for the main study in Miyagi Prefecture (Fig. 1). In this adjunct study, the participants in the resident registers of 4 local governments, Kesennuma, Minamisanriku, Onagawa, and Ishinomaki, were further recruited to participate in the adjunct study when they were in the first trimester. The participants were asked to provide written informed consent for the JECS main study, and next for the adjunct study. Although the JECS recruitment period was from January 2011 until March 2014 for the main study, it was from December 2011 until October 2013 for the adjunct study. The eligibility criteria of the adjunct study were the same as for JECS: (1) residence in the study areas, (2) expected delivery date between 1 August 2011 and mid-2014, 


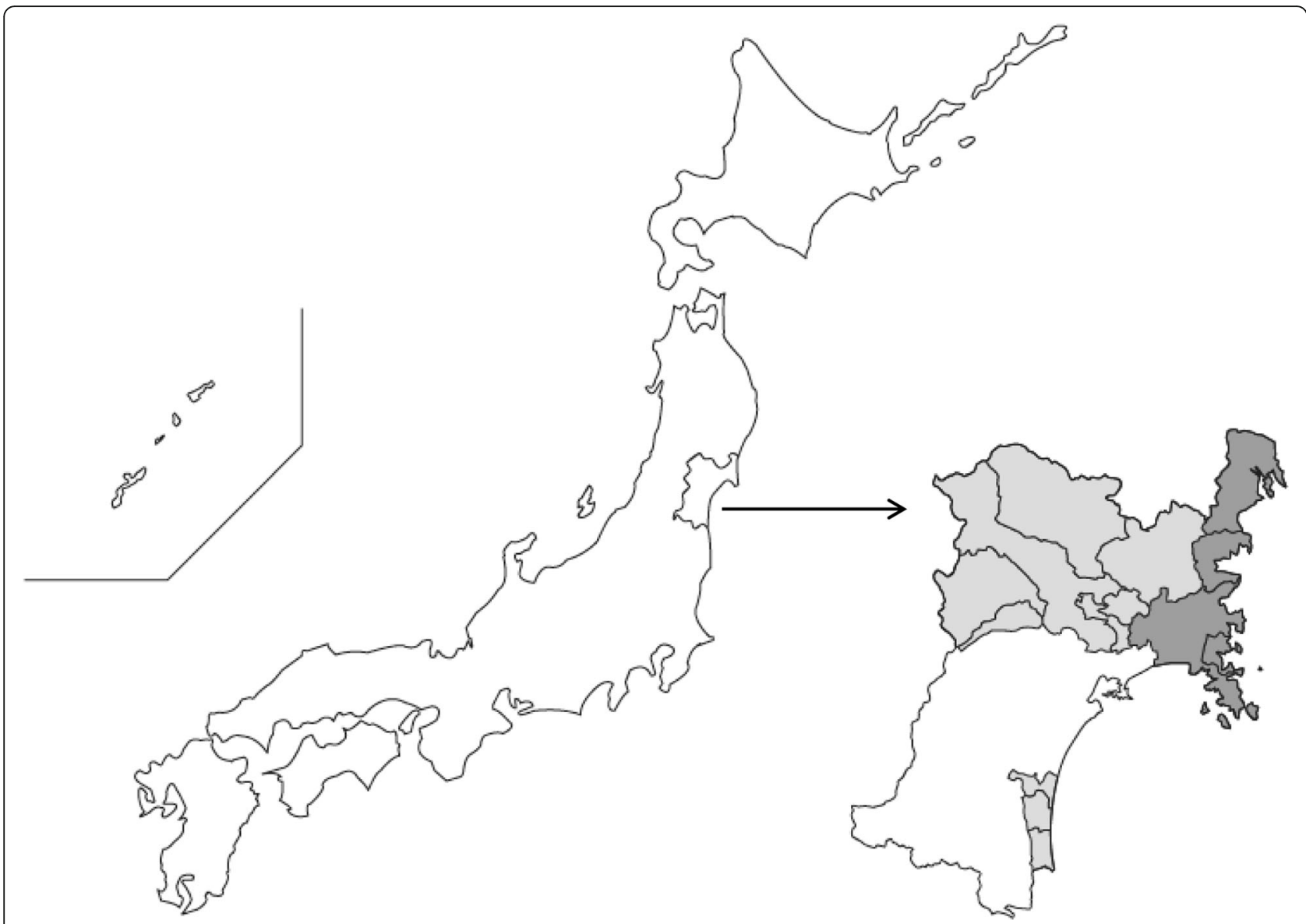

Fig. 1 Study area of JECS (light gray) and of the adjunct study (dark gray) in Miyagi Prefecture

and (3) capable of participating in the study without difficulty [27].

Single mutation and/or genomewide analyses were planned in JECS, and the storage of biological samples for genetic analyses was included in the informed consent [27], though genetic analysis itself was not originally included in the informed consent. Therefore, the participants in this adjunct study were further asked after delivery to provide written informed content for the analysis of single nucleotide polymorphisms, including desaturation and elongation of PUFA, using stored maternal and cord blood. Both JECS and this adjunct study were approved by the Ethics Committee of the Tohoku University Graduate School of Medicine.

\section{Study protocol}

During pregnancy, self-administrated questionnaires were distributed to the pregnant women in their first and second trimesters, and at delivery in JECS [27, 28]. Questionnaires were also distributed to each family periodically after delivery. The JECS questionnaires during pregnancy included all information on basic characteristics and others, including questions about food frequencies, dietary supplement intakes, and physical activities using the short version of the International Physical Activity Questionnaire. The details of the questionnaires were described elsewhere [28]. Medical information was obtained from maternal and infant medical records by JECS, and the collected data were provided to this adjunct study through the data management system of JECS.

Additional procedures for the adjunct study were added to JECS only when they did not disturb the main study. We added the Center for Epidemiological Studies Depression (CES-D) scale to the questionnaire of the second trimester for screening depression. Maternal IQ was evaluated using the Raven standard progressive matrices [15] when the children were aged 7 months. We also added several neurobehavioral assessments for measurement of child neurobehavioral development [29]. These included the neonatal behavioral assessment scale at birth, Kyoto Scale of Psychological Development 2001 at 7 and 24 months, and Kaufman Assessment Battery for Children (K-ABC) at 42 months. Furthermore, the behaviors related to symptoms of attention deficit/hyperactivity disorder (ADHD) were also measured using teacher-rated scales when the children were in kindergarten or nursery school. 


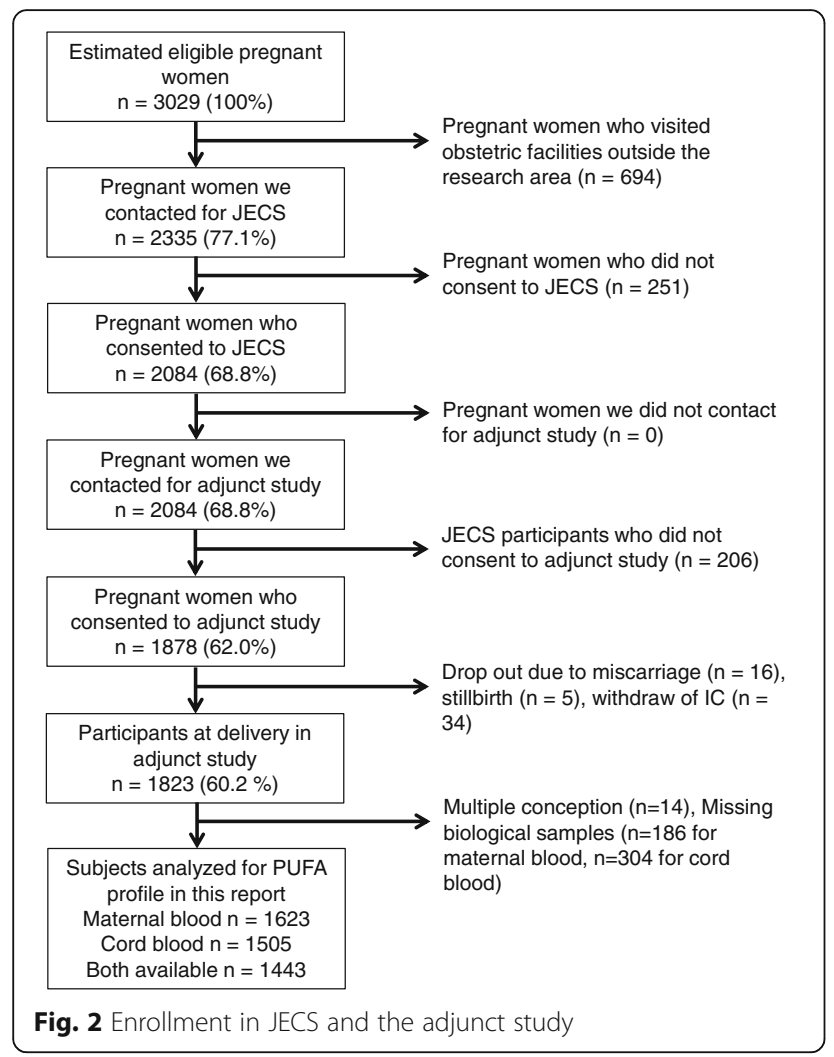

Maternal blood samples were obtained from the mothers at 24-30 weeks of gestation without fasting. Umbilical cord blood samples were obtained from the umbilical cord vein at birth. A vacuum sampling tube with EDTA-2Na as an anticoagulant was used for the blood sampling. These blood samples were transferred to the laboratory within 3 days at $4{ }^{\circ} \mathrm{C}$, and centrifuged at $1600 \mathrm{~g}$ for $10 \mathrm{~min}$ to separate plasma and erythrocytes. The cells were washed twice with physiological saline, and the buffy coat was removed. The plasma and washed erythrocytes were stored at $-80{ }^{\circ} \mathrm{C}$ until fatty acid determination. These procedures did not affect the concentrations of fatty acids [30].

\section{Analysis of erythrocyte fatty acid composition}

Total erythrocyte lipid was extracted using isopropanol/ chloroform according to the method of Rose and Oklander [31]. Briefly, distilled water with $0.1 \mathrm{mM}$ EDTA-2Na was added to the cells and mixed. After $15 \mathrm{~min}$, isopropanol was added slowly with mixing and allowed to stand for $1 \mathrm{~h}$. After chloroform was added and mixed for $1 \mathrm{~h}$, the lipid was transferred into chloroform-methanol 2:1 $(\mathrm{v} / \mathrm{v})$ and washed with distilled water with $0.9 \% \mathrm{KCl}$. After centrifugation at $1,600 \times \mathrm{g}$ for $5 \mathrm{~min}$, the lipid extract obtained in the lower phase was methylated using methanol hydrochloride, and then gas-liquid chromatographic analysis of the fatty acid methyl ester compositions was conducted, as described previously [32].

\section{Statistical analyses}

We obtained information from medical records and/or self-administred questionnaires. The medical record data were used primarily, whereas the information collected through questionnaire was used only as a fallback measure. The information included maternal age at blood collection, marital status (married/not married), educational background ( $<13$ years/longer), household income (5 ranks), living together with parents $(\mathrm{Y} / \mathrm{N})$, smoking habits (never smoker/ex-smokers who quit before pregnancy confirmation/ex-smokers who quit after pregnancy confirmation/smokers during pregnancy), passive smoking $(\mathrm{Y} / \mathrm{N})$, alcohol consumption $(\mathrm{Y} / \mathrm{N})$, history of obstetrical/ gynecological diseases, height and weight before pregnancy, parity (primiparae/multiparae), infertility treatment $(\mathrm{Y} / \mathrm{N})$, gestational age at blood collection and at birth, the child's sex, type of delivery (transvaginal/caesarean section), and birth weight and height. Regarding the smoking habit, the same questionnaire was administered in both the first and second trimesters. We identified the participants as smokers or ex-smokers when they responded to the smoking question in either of the two questionnaires. Alcohol consumption status was divided into two categories in the questionnaire for the second trimester: nondrinkers (i.e., non-drinkers and ex-drinkers who quit drinking before or after confirmation of pregnancy) and drinkers (i.e., participants who continued drinking after confirmation of pregnancy).

In the FFQ, the subjects gave the frequency and portions of consumption for each item over the previous one month during the second trimester. The amounts of consumption were calculated from the frequencies and the portions given by the subjects individually; fish and seafood consumption was from the sum of 21 items, and meat consumption from that of 19 items.

The normality of continuous data was examined using the Shapiro-Wilk test. Since large skewed distributions were observed in the EPA content for maternal blood $($ skewness $=1.81)$ and cord blood (skewness $=3.48)$, EPA was used for analysis after logarithmic transformation. Intakes of fish and meat were also used for analysis after logarithmic transformation. The differences of basic characteristics between the participants of this adjunct study and the non-participants were analyzed using analysis of variance, the chi-square test, and the Fisher exact probability test.

For analysis of the determinants of PUFA profiles, those who had diabetes mellitus and gestational diabetes mellitus were excluded from further analyses because these pathological conditions might affect the metabolism and transport of PUFA between the placenta and fetal 
Table 1 Basic characteristics of the participants and the non-participants having live birth in the adjunct study of JECS

\begin{tabular}{|c|c|c|c|c|c|c|c|}
\hline \multirow[t]{2}{*}{ Variables } & \multicolumn{2}{|c|}{ All JECS Participants } & \multicolumn{5}{|c|}{ Participants of adjunct study Non-participants } \\
\hline & \multicolumn{2}{|c|}{$\bar{n}$} & \multicolumn{2}{|l|}{$n$} & \multicolumn{2}{|l|}{$n$} & \multirow[t]{2}{*}{$p$} \\
\hline Mothers with live birth & 8873 & & 1823 & & 7050 & & \\
\hline Age at delivery (yrs) & 8823 & $30.7 \pm 5.1$ & 1817 & $31.0 \pm 5.3$ & 7006 & $30.6 \pm 5.0$ & 0.0022 \\
\hline Marital status at registration (Married, \%) & 8798 & 94.7 & 1807 & 94.3 & 6991 & 94.8 & 0.39 \\
\hline Maternal education (<13 yrs, \%) & 8746 & 52.9 & 1790 & 54.1 & 6956 & 52.3 & 0.27 \\
\hline Paternal education (<13 yrs, \%) & 8695 & 59.3 & 1778 & 62.9 & 6917 & 59.6 & 0.013 \\
\hline \multicolumn{8}{|l|}{ Household income (million Japanese Yen) (\%) } \\
\hline$<2$ & 575 & 7.4 & 121 & 7.4 & 454 & 7.4 & 0.15 \\
\hline 2 to $<4$ & 3111 & 39.9 & 694 & 42.6 & 2417 & 39.2 & \\
\hline 4 to $<6$ & 2290 & 29.4 & 454 & 27.8 & 1836 & 29.8 & \\
\hline 6 to $<8$ & 972 & 12.5 & 205 & 12.6 & 767 & 12.5 & \\
\hline$\geq 8$ & 842 & 10.8 & 157 & 9.6 & 685 & 11.1 & \\
\hline Living together with parents (Yes, \%) & 8835 & 46.7 & 1818 & 43.3 & 7017 & 47.5 & 0.0012 \\
\hline \multicolumn{8}{|l|}{ Smoking habits (\%) } \\
\hline Never smoked & 4324 & 49.0 & 842 & 46.4 & 3482 & 49.6 & 0.034 \\
\hline Ex-smokers who quit before pregnancy & 2099 & 23.8 & 440 & 24.2 & 1659 & 23.6 & \\
\hline Ex-smokers who quit after pregnancy & 1752 & 19.8 & 377 & 20.8 & 1375 & 19.6 & \\
\hline Smokers during early pregnancy & 657 & 7.4 & 157 & 8.6 & 500 & 7.1 & \\
\hline Passive smoking (Yes, \%) & 8850 & 66.4 & 1819 & 69.2 & 7031 & 65.7 & 0.0047 \\
\hline Alcohol consumption (Drinkers, \%) & 8767 & 2.5 & 1791 & 1.7 & 6976 & 2.7 & 0.013 \\
\hline Prepregnancy BMI (kg/m2) & 8872 & $21.8 \pm 3.6$ & 1823 & $21.9 \pm 3.9$ & 7049 & $21.7 \pm 3.6$ & 0.019 \\
\hline Body weight gain at delivery (kg) & 8822 & $10.8 \pm 4.2$ & 1813 & $10.4 \pm 4.2$ & 7009 & $10.9 \pm 4.1$ & $<0.0001$ \\
\hline Physical activity (mets · min/week) & 8766 & $480(0-18400)$ & 1792 & $480(0-18400)$ & 6974 & $480(0-16400)$ & 0.81 \\
\hline \multicolumn{8}{|l|}{ Obstetrical/gynecological disease (\%) } \\
\hline Diabetes mellitus & 8866 & 0.73 & 1822 & 0.49 & 7044 & 0.80 & 0.16 \\
\hline Gestational diabetes mellitus & 8866 & 1.5 & 1822 & 1.1 & 7044 & 1.6 & 0.11 \\
\hline Pregnancy-induce hypertension & 8866 & 1.1 & 1822 & 2.4 & 7044 & 0.82 & $<0.0001$ \\
\hline Hyperthyroidism & 8866 & 0.43 & 1822 & 0.27 & 7044 & 0.47 & 0.23 \\
\hline Intrauterine growth restriction & 8866 & 1.2 & 1822 & 1.4 & 7044 & 1.2 & 0.37 \\
\hline Placenta abruption & 8866 & 0.20 & 1822 & 0.0 & 7044 & 0.26 & $0.035^{\mathrm{a}}$ \\
\hline Parity (Primipara, \%) & 8783 & 39.4 & 1789 & 37.8 & 6994 & 39.8 & 0.12 \\
\hline Infertility treatment (Yes, \%) & 8814 & 4.6 & 1812 & 5.9 & 7002 & 4.3 & 0.0059 \\
\hline Fish intake (g/day) & 8757 & $29.1(0.8-919)$ & 1788 & $178828.3(1.2-612)$ & 6969 & $29.4(0.8-919)$ & 0.11 \\
\hline Meat intake (g/day) & 8762 & $54.6(0.4-1740)$ & 1789 & $55.5(0.4-1581)$ & 6973 & $54.3(1.5-1740)$ & 0.21 \\
\hline \multicolumn{8}{|l|}{ Supplemental use (\%) } \\
\hline Any kind & 8774 & 38.8 & 1793 & 34.2 & 6981 & 40.0 & $<0.0001$ \\
\hline EPA and/or DHA & 8773 & 2.2 & 1792 & 2.5 & 5981 & 2.1 & 0.30 \\
\hline Breakfast skipper (Skipper, \%) & 8756 & 13.8 & 1788 & 14.8 & 6968 & 13.5 & 0.14 \\
\hline Housewife (Yes, \%) & 8742 & 44.0 & 1787 & 50.6 & 6955 & 42.3 & $<0.0001$ \\
\hline \multicolumn{8}{|l|}{ Number of live births } \\
\hline Singleton births (\%) & 8873 & 99.2 & 1823 & 99.2 & 7050 & 99.1 & 0.69 \\
\hline Gestational age at birth (day) & 8857 & $274.1 \pm 11.8$ & 1819 & $273.6 \pm 13.5$ & 7038 & $274.3 \pm 11.3$ & 0.025 \\
\hline Preterm births $(<37)(\%)$ & 8857 & 5.5 & 1819 & 5.4 & 7038 & 5.5 & 0.93 \\
\hline Sex (Male, \%) & 8865 & 51.0 & 1822 & 51.6 & 7043 & 50.9 & 0.35 \\
\hline
\end{tabular}


Table 1 Basic characteristics of the participants and the non-participants having live birth in the adjunct study of JECS (Continued)

\begin{tabular}{lllllllc}
\hline Type of delivery (Transvaginal, \%) & 8847 & 82.2 & 1819 & 81.9 & 7028 & 82.2 & 0.74 \\
Birth weight (g, Singleton births) & & & & & & 7032 & $3050 \pm 431$ \\
All & 8850 & $3046 \pm 441$ & 1818 & $3031 \pm 479$ & 3580 & $3089 \pm 433$ \\
Male & 4481 & $3095 \pm 433$ & 939 & $3076 \pm 485$ & 0.43 \\
Female & 4289 & $3010 \pm 427$ & 876 & $2984 \pm 468$ & 3450 & $3008 \pm 424$ & 0.15 \\
Low birth weight (<2500 g) (\%) & 8850 & 8.2 & 1818 & 9.8 & 7032 & 7.8 \\
\hline
\end{tabular}

Mean \pm SD or median (min-max)

${ }^{\mathrm{a}}$ Fisher exact test

blood $[4,18,19]$. Those who had pregnancy-induced hypertension, hyperthyroidism, intrauterine growth restriction, and placental abruption were also excluded because of possible associations with delayed fetal development. Univariate associations between PUFA and other variables were examined using Pearson product-moment correlation coefficients (for continuous data) and ANOVA (for categorical data) as appropriate. The variables that were statistically significant in the univariate analysis at the $p<0.05$ level were entered into exploratory multiple stepwise regression analyses. Other factors with strong a priori assumptions (e.g., a maternal smoking habit [33] and physical activity [21]) based on the findings of previous reports were also considered in the analysis of maternal blood. The Akaike information criteria were used in stepwise forward selection for determination of important factors. Participants with missing data for exposure variables were excluded. Variables were tested for collinearity with the variance inflation factor (VIF). All analyses, with two-sided $p$ values, were carried out using the JMP software package version $7 \cdot 0$ (SAS Institute Inc., Cary, NC, USA).

\section{Results}

\section{Registration and basic characteristics}

Of the 9,217 JECS participants, 1,878 pregnant women joined in the adjunct study (Fig. 2). Since the number of maternal and child health handbooks issued by the local governments was 3,029 during the period of enrollment in the adjunct study, the coverage rate was approximately $62 \%$. The numbers of participants who were included in the determination of factors influencing the PUFA profiles were 1,623 for maternal blood, and 1,505 for cord blood.

Characteristics of the mothers and their babies are presented in Table 1. The participants in the adjunct study were slightly older, and the rate of housewifes was higher than for the non-participants. More mothers who participated smoked, skipped breakfast, used some kind of supplementation, and were exposed to environmental tobacco smoke. Prepregnancy BMI, maternal body weight gain at delivery, gestational age and the number of alcohol drinkers were smaller among the participants. The percentage of low-birth-weight newborns $(<2,500 \mathrm{~g})$ was
$9.8 \%$ in the participants in comparison with $7.8 \%$ in non-participants.

\section{PUFA status}

The percentages by weight of fatty acids in maternal and cord blood erythrocytes are shown in Table 2. The DHA level was $7.4 \%$ in maternal blood erythrocytes, which roughly corresponded to the DHA levels that were previously reported in the general Japanese population [21, 26], and was higher than reported for Western countries $[4,20]$.

\section{Determinants of PUFA profiles}

The univariate associations of DHA, EPA, and ARA in maternal and cord blood with basic characteristics are shown in Table 3 for continuous data and Table 4 for categorical data. Maternal erythrocyte DHA and EPA

Table 2 Percentages by weight of fatty acid in maternal and cord blood erythrocytes

\begin{tabular}{|c|c|c|c|}
\hline \multicolumn{2}{|l|}{ Fatty acid } & Maternal blood $(n=1623)$ & Cord blood $(n=1505)$ \\
\hline $14: 0$ & & $0.4(0.2-1.2)$ & $0.4(0.0-0.9)$ \\
\hline $15: 0$ & & $0.1(0.0-0.5)$ & $0.1(0.0-0.3)$ \\
\hline $16: 0$ & & $24.7(20.4-30.4)$ & $27.0(23.1-32.3)$ \\
\hline $16: 1 n-7$ & & $0.3(0.0-1.0)$ & $0.4(0.0-1.9)$ \\
\hline $18: 0$ & & $15.7(12.5-25.2)$ & $18.0(14.7-34.6)$ \\
\hline $18: 1 n-7$ & & $1.1(0.0-1.7)$ & $1.6(0.1-2.3)$ \\
\hline $18: 1 n-9$ & & $12.8(9.0-18.3)$ & $10.0(6.4-17.6)$ \\
\hline $18: 2 n-6$ & LA & $9.1(4.2-14.0)$ & $4.2(2.6-9.6)$ \\
\hline $18: 3 n-3$ & ALA & $0.2(0.0-2.7)$ & $0.0(0.0-0.6)$ \\
\hline $20: 3 n-6$ & & $1.3(0.7-2.5)$ & $2.4(1.2-4.2)$ \\
\hline $20: 4 n-6$ & ARA & $11.6(6.6-16.2)$ & $15.5(9.1-20.0)$ \\
\hline $20: 5 n-3$ & EPA & $0.7(0.1-4.1)$ & $0.3(0.0-2.5)$ \\
\hline $22: 0$ & & $1.2(0.0-9.8)$ & $1.0(0.0-3.3)$ \\
\hline $22: 4 n-6$ & & $2.0(0.2-4.3)$ & $2.9(0.8-5.2)$ \\
\hline $22: 5 n-3$ & & $1.9(0.9-3.5)$ & $0.7(0.3-1.9)$ \\
\hline $22: 6 n-3$ & DHA & $7.4(3.2-11.0)$ & $6.8(3.1-10.7)$ \\
\hline $24: 0$ & & $3.3(0.9-6.8)$ & $3.8(2.9-5.5)$ \\
\hline $24: 1 n-9$ & & $4.1(0.9-6.2)$ & $2.9(0.7-7.0)$ \\
\hline
\end{tabular}

Median (min-max) 
Table 3 Univariate associations between basic characteristics and PUFA levels in maternal and cord blood erythrocyte (Continuous data)

\begin{tabular}{|c|c|c|c|c|c|c|c|c|}
\hline \multirow[t]{2}{*}{ Variables } & \multicolumn{4}{|c|}{ Maternal blood } & \multicolumn{4}{|c|}{ Cord blood } \\
\hline & $n$ & $\mathrm{DHA}$ & $\log -\mathrm{EPA}$ & ARA & $\mathrm{n}$ & $\mathrm{DHA}$ & $\log -E P A$ & ARA \\
\hline Age at blood collection & 1524 & $0.157(<.0001)$ & $0.151(<.0001)$ & $-0.113(<.0001)$ & - & - & - & - \\
\hline Age at delivery & - & - & - & - & 1439 & $0.048(0.068)$ & $0.083(0.0016)$ & $-0.068(0.010)$ \\
\hline Prepregnancy BMl & 1547 & $-0.033(0.19)$ & $-0.014(0.59)$ & $-0.019(0.46)$ & 1442 & $0.002(0.93)$ & $0.009(0.72)$ & $0.003(0.92)$ \\
\hline Body weight gain at blood collection & 1544 & $0.022(0.39)$ & $0.068(0.0076)$ & $-0.058(0.023)$ & - & - & - & - \\
\hline Body weight gain at delivery & - & - & - & - & 1439 & $0.097(0.0002)$ & $0.033(0.21)$ & $-0.028(0.29)$ \\
\hline Gestational age at blood collection & 1544 & $0.005(0.85)$ & $0.0043(0.87)$ & $-0.013(0.61)$ & & & & \\
\hline Gestational age at birth & - & - & - & - & 1442 & $0.183(<.0001)$ & $0.005(0.86)$ & $-0.14(<.0001)$ \\
\hline Physical activity & 1528 & $-0.021(0.42)$ & $-0.049(0.056)$ & $0.037(0.16)$ & 1432 & $-0.017(0.53)$ & $-0.025(0.34)$ & $0.034(0.20)$ \\
\hline Fish intake & 1524 & $0.186(<.0001)$ & $0.233(<.0001)$ & $-0.149(<.0001)$ & 1428 & $0.109(<.0001)$ & $0.171(<.0001)$ & $-0.135(<.0001)$ \\
\hline Meat intake & 1525 & $-0.040(0.12)$ & $-0.031(0.23)$ & $0.072(0.0052)$ & 1429 & $-0.043(0.10)$ & $-0.048(0.070)$ & $0.044(0.87)$ \\
\hline Each PUFA in maternal blood & - & - & - & - & 1442 & $0.349(<.0001)$ & $0.625(<.0001)$ & $0.334(<.0001)$ \\
\hline
\end{tabular}

Examined with Pearson correlation, $r(p)$

levels were weakly correlated with maternal age and fish intake, whereas maternal ARA was inversely correlated with these factors, and positively correlated with meat intake. Maternal DHA and EPA were also associated with maternal education, household income, smoking, and passive smoking. The fatty acids in cord blood showed a high correlation with the corresponding fatty acids in maternal blood. Furthermore, cord blood erythrocyte EPA showed a similar association to that of maternal EPA. Cord blood DHA had a positive correlation with body weight gain during pregnancy, gestational age, and fish intake, whereas cord blood erythrocyte ARA was inversely correlated with gestational age and fish intake.

Variables having significant associations in these univariate analyses and some other factors such as a maternal smoking habit and physical activity were included in multiple stepwise regression analyses. The best regression models are shown in Table 5 for maternal blood and Table 6 for cord blood. Maternal erythrocyte DHA was associated positively with fish intake and maternal age, and this fatty acid was decreased in smokers. Maternal EPA was associated positively with fish intake, maternal body weight gain, and age. This fatty acid increased in the autumn and in the participants having the highest income and higher educational level. In contrast, maternal ARA was associated inversely with fish intake, body weight gain, and maternal age. Meat intake was also associated positively with maternal ARA. In cord blood analyses, all fatty acids were potently associated with corresponding fatty acids in maternal blood. Cord blood DHA was associated with maternal body weight gain and gestational age, and the level of this fatty acid was higher in male newborns. However, there was no association between cord blood DHA content and maternal fish intake. In contrast, cord blood EPA was positively associated with fish intake, and a higher concentration was observed in the autumn. The cord blood ARA concentration was higher in female newborns and in mothers who skipped breakfast. This fatty acid was negatively associated with gestational age and fish intake.

\section{Discussion}

In the present report, we described the protocol and the characteristics of the participants in a birth cohort study to examine the beneficial effects of n-3 PUFA on maternal health and child development. The number of participants in the adjunct study was sufficient for the above purposes. By adding several measurements to JECS, maternal health and child development could be observed in this adjunct study. In particular, the KSPD and K$A B C$ were expected to be useful to examine child development. The KSPD seems to be equivalent to the Bayley Scale of Child Development, which has been utilized in many epidemiological studies. The K-ABC has been used to demonstrate the beneficial effects of fish oil supplementation during pregnancy and lactation on child intelligence at the age of 4 years [1] and the adverse effects of prenatal PCB exposure in Japan [15]. Furthermore, this is the first study that connects an environmental measurement system including methylmercury and POPs that may be intaken through fish consumption. These unique features thereby can help us to examine the risks and benefits of fish consumption by comparing the effects of PUFA status and toxicant exposure on child development.

In the baseline data of this birth cohort study, we searched for dietary, anthropometric, and lifestyle determinants of DHA, EPA and ARA content in erythrocytes of maternal blood and cord blood. As expected, fish consumption was the primary determinant to increase the contents of DHA and EPA, and to decrease that of ARA 


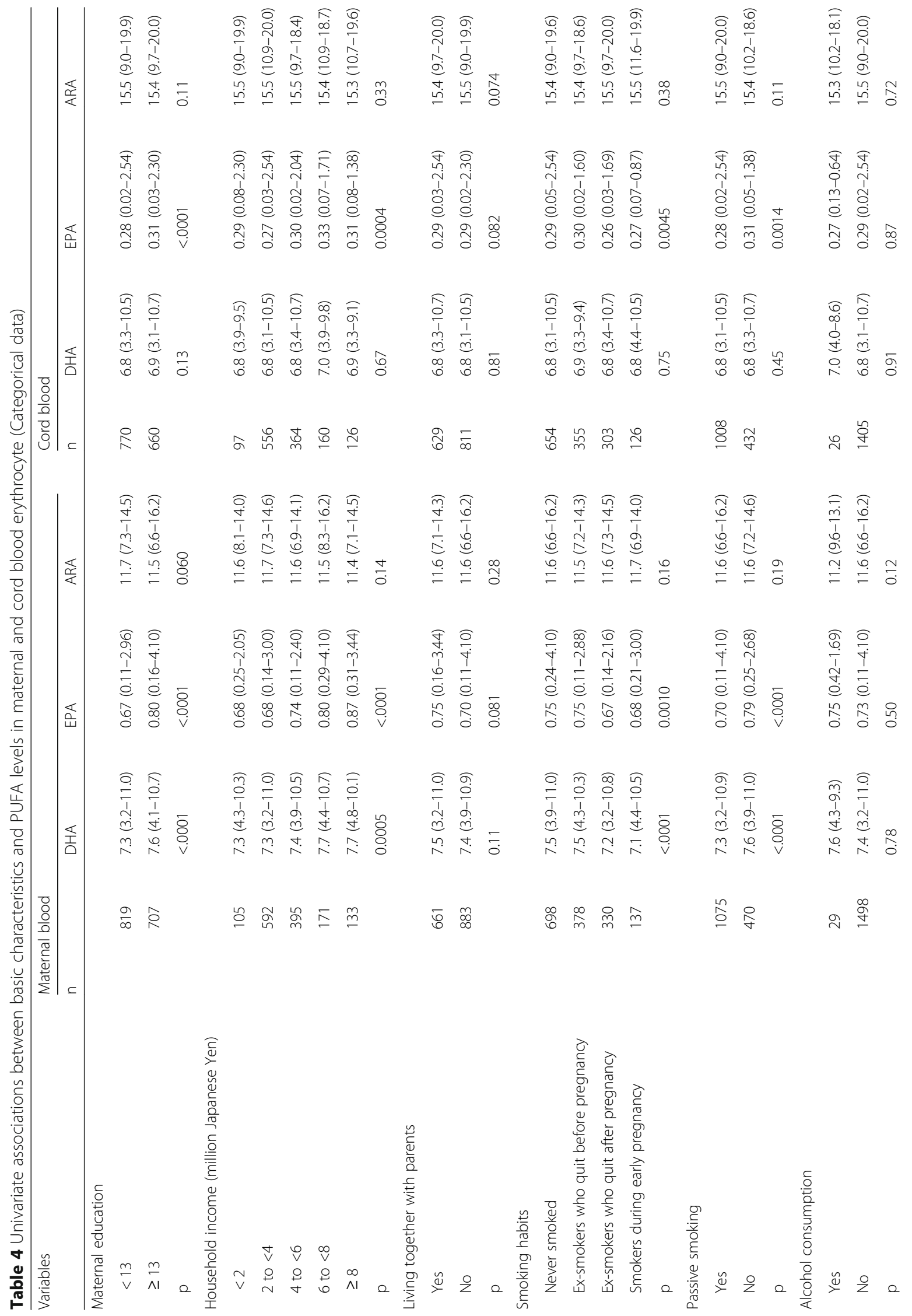




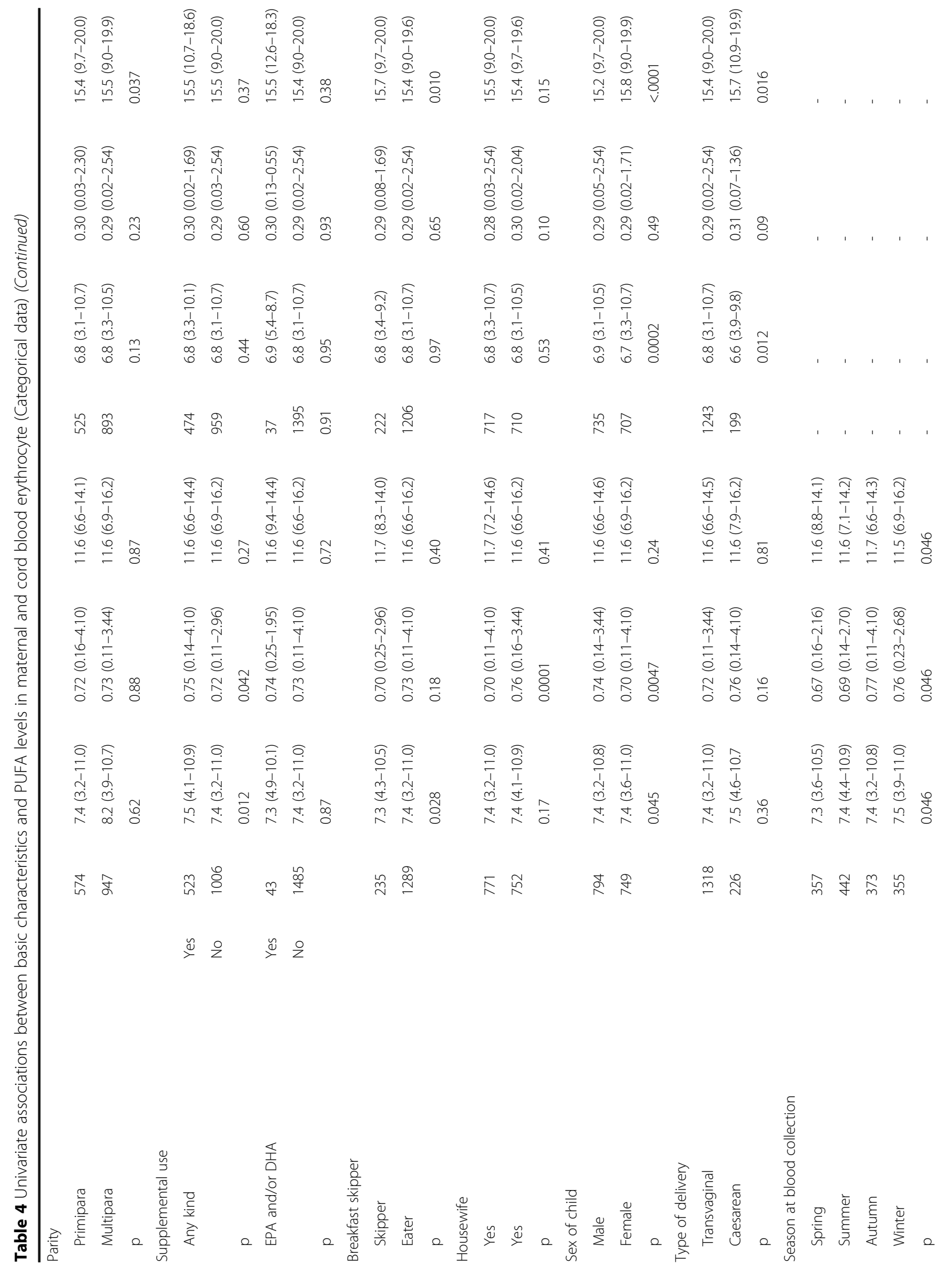




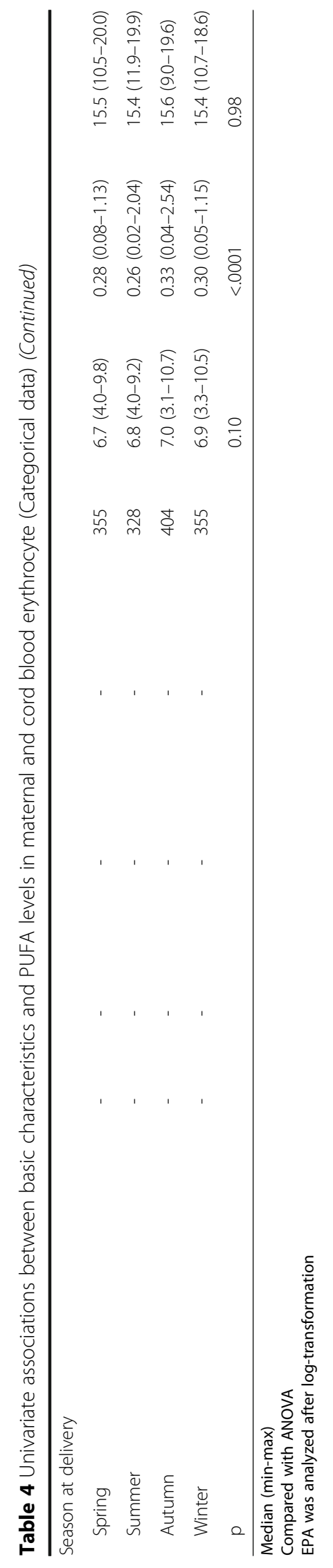


Table 5 Multiple stepwise regression analyses for determinants of PUFA levels in maternal blood erythrocytes

\begin{tabular}{|c|c|c|c|c|c|c|c|c|c|}
\hline \multirow{3}{*}{$\begin{array}{l}\text { Adjusted model } \mathrm{R}^{2} \\
\text { Variables }\end{array}$} & \multicolumn{3}{|c|}{$\mathrm{DHA}(n=1376)$} & \multicolumn{3}{|c|}{$\log -\mathrm{EPA}(n=1374)$} & \multicolumn{3}{|c|}{ ARA $(n=1504)$} \\
\hline & \multicolumn{3}{|l|}{0.072} & \multicolumn{3}{|l|}{0.122} & \multicolumn{3}{|l|}{0.056} \\
\hline & B & Standardized $\beta$ & $\mathrm{p}$ & B & Standardized $\beta$ & $\mathrm{p}$ & B & Standardized $\beta$ & $\mathrm{p}$ \\
\hline Maternal age at blood collection & 0.025 & 0.12 & $<.0001$ & 0.0034 & 0.090 & 0.0007 & -0.020 & -0.089 & 0.0005 \\
\hline Maternal education (<13 yrs) & 0.056 & 0.049 & 0.078 & 0.019 & 0.10 & 0.0005 & - & & \\
\hline \multicolumn{10}{|l|}{ Household income } \\
\hline <2 (million Japanese Yen) & \multicolumn{3}{|c|}{ reference } & \multicolumn{3}{|c|}{ reference } & \multicolumn{3}{|l|}{-} \\
\hline 2 to $<4$ & -0.084 & -0.046 & 0.098 & -0.021 & -0.066 & 0.014 & - & & \\
\hline 4 to $<6$ & -0.092 & -0.046 & 0.11 & -0.013 & -0.034 & 0.19 & - & & \\
\hline 6 to $<8$ & 0.11 & 0.042 & 0.16 & 0.0079 & 0.018 & 0.54 & - & & \\
\hline$\geq 8$ & 0.073 & 0.027 & 0.38 & 0.041 & 0.086 & 0.0034 & - & & \\
\hline \multicolumn{10}{|l|}{ Smoking habits } \\
\hline Never smoked & \multicolumn{3}{|c|}{ reference } & \multicolumn{3}{|c|}{ reference } & \multicolumn{3}{|l|}{-} \\
\hline Ex-smokers before pregnancy & -0.084 & 0.088 & 0.10 & 0.014 & 0.059 & 0.13 & - & & \\
\hline Ex-smokers after pregnancy & -0.070 & -0.048 & 0.23 & -0.010 & -0.038 & 0.34 & - & & \\
\hline Smokers & -0.17 & -0.10 & 0.032 & -0.015 & -0.049 & 0.27 & - & & \\
\hline Body weight gain & - & & & 0.0057 & 0.091 & 0.0004 & -0.027 & -0.072 & 0.0044 \\
\hline Fish intake & 0.43 & 0.16 & $<.0001$ & 0.094 & 0.20 & $<.0001$ & -0.57 & -0.21 & $<.0001$ \\
\hline Meat intake & - & & & - & & & 0.58 & 0.17 & $<.0001$ \\
\hline Sex (male) & 0.055 & 0.049 & 0.061 & 0.0091 & 0.046 & 0.068 & - & & \\
\hline \multicolumn{10}{|l|}{ Season at blood collection } \\
\hline Spring & \multicolumn{3}{|c|}{ reference } & \multicolumn{3}{|c|}{ reference } & \multicolumn{3}{|l|}{-} \\
\hline Summer & -0.067 & -0.043 & 0.17 & -0.021 & -0.076 & 0.012 & - & & \\
\hline Autumn & 0.051 & 0.031 & 0.32 & 0.032 & 0.11 & 0.0003 & - & & \\
\hline Winter & 0.087 & 0.052 & 0.097 & 0.028 & 0.097 & 0.0017 & - & & \\
\hline
\end{tabular}

Variables included in the model are indicated

in maternal blood. Similarly, fish consumption also increased EPA content, and decreased ARA content in cord blood, however, it did not affect the DHA content in cord blood. Although the explanation of this difference is not known, DHA incorporation into erythrocyte membrane is slow in individuals with higher DHA content, and it is supposed that erythrocyte membrane DHA content might be regulated [34]. A similar regulation may affect the transfer of DHA across the placenta. While it was reported that fish consumption increased in the elder population in Japan [21], age was still an independent determinant for all fatty acids even after the adjustment of covariates as reported previously [19, 35]. The effect of maternal smoking on the level of DHA in maternal red blood cells is controversial. Studies of pregnant German women [18] and schizophrenic patients [2] showed that smoking status predicted lower levels of DHA, but other studies did not confirm the effect of smoking $[19,21]$. In the present study, maternal smoking was found to decrease the content of DHA and EPA in maternal blood. One possible explanation is that nonsmokers ate more oily fishes than smokers [36]. Other possibilities included alteration in the metabolism and synthesis of fatty acids and oxidative destruction of highly unsaturated fatty acids after exposure to cigarette smoke. Interestingly, though, maternal smoking had no influence on PUFA profiles in cord blood in the present study. Seasonal changes in the content of EPA were observed both in maternal blood and cord blood. Increased consumption of oily fishes during autumn was probably related to the increased level of EPA. Similarly, the participants with higher household incomes had higher EPA content in maternal blood, suggesting a difference in the kind of consumed fishes dependent on the economic situation. Supplemental use of PUFA had no effect on the contents in maternal blood. Although the exact dose of PUFA supplementation was not inquired about in the questionnaire, a possible explanation is that the dose and frequency of PUFA supplementation might be too small. These above inferences must be evaluated through future studies including a quantitative FFQ. While a Japanese study indicated that physical activity was inversely related to the concentrations of DHA and EPA in the general population [21], physical activity was not 
Table 6 Multiple stepwise regression analyses for determinants of PUFA levels in cord blood erythrocytes

\begin{tabular}{|c|c|c|c|c|c|c|c|c|c|}
\hline \multirow{3}{*}{$\begin{array}{l}\text { Adjusted model } R^{2} \\
\text { Variables }\end{array}$} & \multicolumn{3}{|c|}{$\mathrm{DHA}(n=1379)$} & \multicolumn{3}{|c|}{$\log$-EPA $(n=1247)$} & \multicolumn{3}{|c|}{ ARA $(n=1365)$} \\
\hline & \multicolumn{3}{|l|}{0.165} & \multicolumn{3}{|l|}{0.348} & \multicolumn{3}{|l|}{0.171} \\
\hline & $B$ & Standardized $\beta$ & $\mathrm{p}$ & $\mathrm{B}$ & Standardized $\beta$ & $\mathrm{p}$ & $\mathrm{B}$ & Standardized $\beta$ & $p$ \\
\hline PUFA level in maternal blood & 0.31 & 0.34 & $<.0001$ & 0.32 & 0.54 & $<.0001$ & 0.39 & 0.31 & $<.0001$ \\
\hline \multicolumn{10}{|l|}{ Household income } \\
\hline <2 (million Japanese Yen) & - & & & \multicolumn{3}{|c|}{ reference } & \multicolumn{3}{|l|}{-} \\
\hline 2 to $<4$ & \multicolumn{3}{|l|}{-} & -0.022 & -0.058 & 0.017 & \multicolumn{3}{|l|}{-} \\
\hline 4 to $<6$ & \multicolumn{3}{|l|}{-} & 0.008 & 0.019 & 0.45 & \multicolumn{3}{|l|}{-} \\
\hline 6 to $<8$ & \multicolumn{3}{|l|}{-} & 0.016 & 0.031 & 0.23 & \multicolumn{3}{|l|}{-} \\
\hline$\geq 8$ & \multicolumn{3}{|l|}{-} & -0.013 & -0.022 & 0.40 & \multicolumn{3}{|l|}{-} \\
\hline \multicolumn{10}{|l|}{ Smoking habits } \\
\hline Never smoked & \multicolumn{3}{|l|}{-} & \multicolumn{3}{|c|}{ reference } & \multicolumn{3}{|l|}{ - } \\
\hline Ex-smokers before pregnancy & \multicolumn{3}{|l|}{-} & 0.006 & 0.020 & 0.56 & \multicolumn{3}{|l|}{-} \\
\hline Ex-smokers after pregnancy & \multicolumn{3}{|l|}{ - } & -0.014 & -0.046 & 0.19 & \multicolumn{3}{|l|}{-} \\
\hline Smokers & \multicolumn{3}{|l|}{-} & -0.003 & -0.007 & 0.86 & \multicolumn{3}{|l|}{-} \\
\hline Body weight gain & 0.016 & 0.064 & 0.011 & - & & & \multicolumn{3}{|l|}{-} \\
\hline Fish intake & \multicolumn{3}{|l|}{-} & 0.036 & 0.065 & 0.0061 & -0.26 & -0.079 & 0.0016 \\
\hline Breakfast skipper (Yes) & \multicolumn{3}{|l|}{-} & \multicolumn{3}{|l|}{-} & 0.13 & 0.065 & 0.0088 \\
\hline Gestational age & 0.020 & 0.18 & $<.0001$ & - & & & -0.024 & -0.15 & $<.0001$ \\
\hline Sex (male) & 0.098 & 0.096 & 0.0001 & - & & & -0.26 & -0.18 & $<.0001$ \\
\hline Season at birth & & & & & & & & & \\
\hline Spring & - & & & referenc & & & - & & \\
\hline Summer & - & & & -0.032 & -0.095 & 0.0008 & - & & \\
\hline Autumn & - & & & 0.073 & 0.22 & $<.0001$ & - & & \\
\hline Winter & - & & & -0.023 & -0.068 & 0.017 & - & & \\
\hline
\end{tabular}

Variables included in the model are indicated

identified as a determinant in pregnant women. Although the maternal ARA level was associated with skipping breakfast, the mechanism of this is currently unknown.

Basically, ARA and DHA are sourced both from the diet and endogenously from desaturation and elongation of their dietary precursors. Furthermore, several SNPs in the desaturase gene cluster affect the proportions of PUFA and long chain PUFA in human blood [26]. Since sex hormones also influence the activity of desaturase and elongase enzymes in women [24], the sex differences observed in the contents of cord blood DHA and ARA in the present study were possibly due in part to hormonal differences between female and male newborns. Genomic and hormonal examinations are currently under investigation in this adjunct study.

The present study has several limitations. First, it was a single-center study that was performed in coastal areas of the Tohoku region, where the fish consumption seems to be high. Second, soon after JECS started in January 2011, the Great East Japan earthquake and subsequent Tohoku tsunami occurred in the research areas on
March 11, 2011. The registration of this adjunct study was started in October 2011; however, the life of most participants might be still influenced by the disaster to a greater or lesser extent. In particular, seafood consumption in these coastal areas might have been temporarily decreased due to harmful rumors about food safety and radionuclide contamination and damage to many harbors and fish markets [37]. In contrast, the strengths of the study include the relatively large number of participants and measurements of fatty acids in biological materials. Furthermore, this study was designed to cooperate with the national JECS study, in which risk analyses for several toxic substances such as POPs, heavy metals, and various other chemical exposures were performed simultaneously [27]. Since some toxicants are ingested through the consumption of seafood [38], this cooperation allowed us to examine the nutritional effects of $n-3$ PUFA while considering exposures to toxic contaminants derived from marine products as mentioned above. To our knowledge, this is the first birth cohort study to examine both the nutritional and toxic aspects of fish consumption in Japan. 


\section{Conclusion}

A birth cohort was established to examine the beneficial effects of PUFAs on maternal health and child development as an adjunct study of JECS. Currently child development is being monitored using several psychological and behavioral approaches. Since this adjunct study connects environmental measurement systems through JECS, this unique feature can thereby be used to examine both the beneficial and risk aspects of fish consumption simultaneously. Based on the baseline data, the determinants of PUFA status in maternal and cord blood were examined and, as expected, maternal fish consumption was a potent factor. Considering that the fish consumption of Japanese has been decreasing over the past decade according to $\mathrm{Na}$ tional Health and Nutrition Surveys, understanding of non-dietary determinants will be of importance to improve the maternal status of PUFA. If the optimum PUFA level during pregnancy can be defined via this birth cohort study, better knowledge of the factors determining the levels of PUFAs in maternal and cord blood will help to improve the PUFA status in mothers and newborns.

\section{Abbreviations}

ADHD: Attention deficit/hyperactivity disorder; ALA: a-linolenic acid; ARA: Arachidonic acid; BMI: Body mass index; CES-D: The center for epidemiological studies depression; DHA: Docosahexaenoic acid; EPA: Eicosapentaenoic acid; JECS: Japan Environment and children's study; K-ABC: Kaufman assessment battery for children; LA: Linoleic acid; MOE: Ministry of the environment; $n-3$ PUFA: N-3 polyunsaturated fatty acids; n-3 PUFA: N-3 polyunsaturated fatty acids; POPs: Persistent organic pollutants; SNPs: Single nucleotide polymorphisms

\section{Acknowledgements}

The authors would like to thank all participants of the study, research coordinators and members of the JECS Miyagi Regional Centre, and personnel at hospitals and clinics that collaborated in the adjunct study.

\section{Funding}

This work was supported by the Environment Research and Technology Development Fund from the Ministry of the Environment, Japan (C-1153 (2011-2013) and 5-1451(2014-2016)) and by JSPS KAKENHI Grants (numbers 26293146 and 26500016).

\section{Availability of data and materials}

It is not possible to share the raw research data publicly since data privacy could be compromised. However, the raw data are available with the corresponding author, and any researcher interested to gain access to the raw data can address his/her request to the corresponding author at the contact information mentioned in the manuscript. All data generated or analyzed during this study are included in this published article and its supplementary information.

\section{Authors' contributions}

All authors contributed to the generation of the paper. SS contributed in designing, implementation of the study, and critically prepared, read and agreed on the final format of the submitted paper. NY is primary investigator of this whole cohort study, and KN is ex-primary investigator, conceived the study idea, and designed the study. TK, FK, and TM carried out biological monitoring. NT, SM, HN, TA, and KN participated in data collection, analysis and study implementation. KT and KY contributed to develop the study protocol in the point of statistical view. Yl, NK and MK supervised the study from the point of exposure science. TA and YK participated in study design and critical review of the final manuscript. All the authors read and accepted the final draft of the manuscript.

\section{Competing interests}

The authors declare that they have no competing interest.

\section{Consent for publication}

Not applicable.

\section{Ethics approval and consent to participate}

Both JECS and this adjunct study were approved by the Ethics Committee of the Tohoku University Graduate School of Medicine. This study was conducted with written informed consent from all subjects.

\section{Publisher's Note}

Springer Nature remains neutral with regard to jurisdictional claims in published maps and institutional affiliations.

\section{Author details}

${ }^{1}$ Department of Gynecology and Obstetrics, Tohoku University Graduate School of Medicine, Sendai, Japan. ${ }^{2}$ Department of Obstetrics and Gynecology, Yamagata Prefectural Central Hospital, Aoyagi 1800, Yamagata 990-2292, Japan. ${ }^{3}$ Faculty of Nutrition, Kagawa Nutrition University, Saitama, Japan. ${ }^{4}$ Department of Development and Environmental Medicine, Tohoku University Graduate School of Medicine, Sendai, Japan. ${ }^{5}$ Food and Biodynamic Chemistry Laboratory, Graduate School of Agricultural Sciences, Tohoku University, Sendai, Japan. ${ }^{6}$ Department of Human Health and Nutrition, Shokei Gakuin University Graduate School, Natori, Japan. ${ }^{7} \mathrm{New}$ Industry Creation Hatchery Center, Tohoku University, Sendai, Japan. ${ }^{8}$ Department of Informative Genetics, Tohoku University Graduate School of Medicine, Sendai, Japan. 'Department of Hygiene, School of Medicine, Wakayama Medical University, Wakayama, Japan. ${ }^{10}$ Department of Occupational and Environmental Health, Nagoya City University Graduate School of Medical Sciences, Nagoya, Japan.

Received: 18 November 2016 Accepted: 8 February 2017

Published online: 04 April 2017

References

1. Helland IB, Smith L, Saarem K, Saugstad OD, Drevon CA. Maternal supplementation with very-long-chain n-3 fatty acids during pregnancy and lactation augments children's IQ at 4 years of age. Pediatrics. 2003;111:e39-44.

2. Hibbeln JR, Davis JM, Steer C, Emmett P, Rogers I, Williams C, et al. Maternal seafood consumption in pregnancy and neurodevelopmental outcomes in childhood (ALSPAC study): an observational cohort study. Lancet. 2007;369:578-85.

3. Oken E, Osterdal ML, Gillman MW, Knudsen VK, Halldorsson TI, Strom M, et al. Associations of maternal fish intake during pregnancy and breastfeeding duration with attainment of developmental milestones in early childhood: a study from the Danish national birth cohort. Am J Clin Nutr. 2008;88:789-96.

4. Hanebutt FL, Demmelmair H, Schiessl B, Larque E, Koletzko B. Long-chain polyunsaturated fatty acid (LC-PUFA) transfer across the placenta. Clin Nutr. 2008:27:685-93.

5. Martinez-Victoria E, Yago MD. Omega 3 polyunsaturated fatty acids and body weight. Br J Nutr. 2012;107(Suppl 2):S107-116.

6. Koletzko B, Boey CC, Campoy C, Carlson SE, Chang N, Guillermo-Tuazon MA et al. Current information and Asian perspectives on long-chain polyunsaturated fatty acids in pregnancy, lactation, and infancy: systematic review and practice recommendations from an early nutrition academy workshop. Ann Nutr Metab. 2014;65:49-80.

7. Innis SM. Metabolic programming of long-term outcomes due to fatty acid nutrition in early life. Matern Child Nutr. 2011;7(Suppl 2):112-23.

8. Hamazaki K, Harauma A, Otaka Y, Moriguchi T, Inadera H. Serum n-3 polyunsaturated fatty acids and psychological distress in early pregnancy: adjunct study of Japan environment and children's study. Transl Psychiatry. 2016:6:e737.

9. Shapiro GD, Fraser WD, Seguin JR. Emerging risk factors for postpartum depression: serotonin transporter genotype and omega-3 fatty acid status. Can J Psychiatry. 2012;57:704-12.

10. Vidakovic AJ, Jaddoe WW, Gishti O, Felix JF, Williams MA, Hofman A, et al. Body mass index, gestational weight gain and fatty acid concentrations during pregnancy: the generation R Study. Eur J Epidemiol. 2015;30:1175-85. 
11. Lauritzen L, Carlson SE. Maternal fatty acid status during pregnancy and lactation and relation to newborn and infant status. Matern Child Nutr. 2011;7(Suppl 2):41-58.

12. Govarts E, Nieuwenhuijsen M, Schoeters G, Ballester F, Bloemen $K$, de Boer M, et al. Birth weight and prenatal exposure to polychlorinated biphenyls (PCBs) and dichlorodiphenyldichloroethylene (DDE): a meta-analysis within 12 European Birth Cohorts. Environ Health Perspect. 2012;120:162-70.

13. Wells EM, Herbstman JB, Lin YH, Jarrett J, Verdon CP, Ward C, et al. Cord blood methylmercury and fetal growth outcomes in Baltimore newborns: potential confounding and effect modification by omega-3 fatty acids, selenium, and sex. Environ Health Perspect. 2016;124:373-9.

14. Grandjean P, Weihe P, Debes F, Choi AL, Budtz-Jorgensen E. Neurotoxicity from prenatal and postnatal exposure to methylmercury. Neurotoxicol Teratol. 2014;43:39-44

15. Tatsuta N, Nakai K, Murata K, Suzuki K, Iwai-Shimada M, Kurokawa N, et al. Impacts of prenatal exposures to polychlorinated biphenyls, methylmercury, and lead on intellectual ability of 42-month-old children in Japan. Environ Res. 2014;133:321-6.

16. Meldrum SJ, D'Vaz N, Casadio Y, Dunstan JA, Niels Krogsgaard-Larsen N, Simmer K, et al. Determinants of DHA levels in early infancy: differential effects of breast milk and direct fish oil supplementation. Prostaglandins Leukot Essent Fatty Acids. 2012;86:233-9.

17. Al MD, van Houwelingen AC, Hornstra G. Relation between birth order and the maternal and neonatal docosahexaenoic acid status. Eur J Clin Nutr. 1997;51:548-53.

18. Gellert S, Schuchardt JP, Hahn A. Higher omega-3 index and DHA status in pregnant women compared to lactating women - Results from a German nation-wide cross-sectional study. Prostaglandins Leukot Essent Fatty Acids. 2016;109:22-8.

19. Sands SA, Reid KJ, Windsor SL, Harris WS. The impact of age, body mass index, and fish intake on the EPA and DHA content of human erythrocytes. Lipids. 2005:40:343-7.

20. Friedman AN, Moe SM, Perkins SM, Li Y, Watkins BA. Fish consumption and omega-3 fatty acid status and determinants in long-term hemodialysis. Am J Kidney Dis. 2006;47:1064-71.

21. Itomura M, Fujioka S, Hamazaki K, Kobayashi K, Nagasawa T, Sawazaki S, et al. Factors influencing EPA + DHA levels in red blood cells in Japan. In vivo. 2008;22:131-5.

22. Cohen BE, Garg SK, Ali S, Harris WS, Whooley MA. Red blood cell docosahexaenoic acid and eicosapentaenoic acid concentrations are positively associated with socioeconomic status in patients with established coronary artery disease: data from the Heart and Soul Study. J Nutr. 2008;138:1135-40.

23. Wagner A, Simon C, Morio B, Dallongeville J, Ruidavets JB, Haas B, et al. Omega-3 index levels and associated factors in a middle-aged French population: the MONA LISA-NUT Study. Eur J Clin Nutr. 2015;69:436-41.

24. Childs CE, Romeu-Nadal M, Burdge GC, Calder PC. Gender differences in the n-3 fatty acid content of tissues. Proc Nutr Soc. 2008;67:19-27.

25. Carnielli VP, Simonato M, Verlato G, Luijendijk I, De Curtis M, Sauer PJ, et al. Synthesis of long-chain polyunsaturated fatty acids in preterm newborns fed formula with long-chain polyunsaturated fatty acids. Am J Clin Nutr. 2007;86:1323-30

26. Horiguchi S, Nakayama K, Iwamoto S, Ishijima A, Minezaki T, Baba M, et al. Associations between a fatty acid desaturase gene polymorphism and blood arachidonic acid compositions in Japanese elderly. Prostaglandins Leukot Essent Fatty Acids. 2016;105:9-14.

27. Kawamoto T, Nitta H, Murata K, Toda E, Tsukamoto N, Hasegawa M, et al. Rationale and study design of the Japan environment and children's study (JECS). BMC Public Health. 2014;14:25.

28. Michikawa T, Nitta H, Nakayama SF, Ono M, Yonemoto J, Tamura K, et al The Japan Environment and Children's Study (JECS): a preliminary report on selected characteristics of approximately 10000 pregnant women recruited during the first year of the study. J Epidemiol. 2015;25:452-8.

29. Nakai K, Suzuki K, Oka T, Murata K, Sakamoto M, Okamura K, et al. The Tohoku Study of Child Development: a cohort study of effects of perinatal exposures to methylmercury and environmentally persistent organic pollutants on neurobehavioral development in Japanese children. Tohoku J Exp Med. 2004;202:227-37.

30. Kawabata T, Nakai K, Hagiwara C, Kurokawa N, Murata K, Yaginuma K, et al. Comparison of long-chain polyunsaturated fatty acids in plasma and erythrocyte phospholipids for biological monitoring. Nihon Eiseigaku Zasshi. 2011;66:108-14. in Japanese.

31. Rose $\mathrm{HG}$, Oklander M. Improved procedure for the extraction of lipids from human erythrocytes. J Lipid Res. 1965;6:428-31.

32. Hirota S, Adachi N, Gomyo T, Kawashima H, Kiso Y, Kawabata T. Low-dose arachidonic acid intake increases erythrocytes and plasma arachidonic acid in young women. Prostaglandins Leukot Essent Fatty Acids. 2010;83:83-8.

33. Simon JA, Fong J, Bernert Jr JT, Browner WS. Relation of smoking and alcohol consumption to serum fatty acids. Am J Epidemiol. 1996;144:325-34.

34. Cao J, Schwichtenberg KA, Hanson NQ, Tsai MY. Incorporation and clearance of omega-3 fatty acids in erythrocyte membranes and plasma phospholipids. Clin Chem. 2006:52:2265-72.

35. Flock MR, Skulas-Ray AC, Harris WS, Etherton TD, Fleming JA, Kris-Etherton PM. Determinants of erythrocyte omega-3 fatty acid content in response to fish oil supplementation: a dose-response randomized controlled trial. J Am Heart Assoc. 2013;2:e000513.

36. Scaglia N, Chatkin J, Chapman KR, Ferreira I, Wagner M, Selby P, et al. The relationship between omega-3 and smoking habit: a cross-sectional study. Lipids Health Dis. 2016;15:61.

37. Tatsuta N, Nakai K, Satoh H, Murata K. Impact of the great east Japan earthquake on child's IQ. J Pediatr. 2015;167:745-51.

38. Suzuki K, Nakai K, Sugawara T, Nakamura T, Ohba T, Shimada M, et al. Neurobehavioral effects of prenatal exposure to methylmercury and PCBs, and seafood intake: neonatal behavioral assessment scale results of Tohoku study of child development. Environ Res. 2010;110:699-704.

\section{Submit your next manuscript to BioMed Central and we will help you at every step:}

- We accept pre-submission inquiries

- Our selector tool helps you to find the most relevant journal

- We provide round the clock customer support

- Convenient online submission

- Thorough peer review

- Inclusion in PubMed and all major indexing services

- Maximum visibility for your research

Submit your manuscript at www.biomedcentral.com/submit
) Biomed Central 